\title{
BMJ Open Efficacy and safety of sodium-glucose cotransporter 2 inhibitors (SGLT-2is) and glucagon-like peptide-1 receptor agonists (GLP-1RAs) in patients with type 2 diabetes: a systematic review and network meta-analysis study protocol
}

\author{
Humaira Hussein, ${ }^{1,2}$ Francesco Zaccardi, ${ }^{2}$ Nafeesa N Dhalwani, ${ }^{2}$ \\ Melanie J Davies, ${ }^{2,3}$ Kamlesh Khunti, ${ }^{2,3}$ Laura J Gray ${ }^{1,2}$
}

To cite: Hussein $\mathrm{H}$, Zaccardi F, Dhalwani NN, et al. Efficacy and safety of sodium-glucose cotransporter 2 inhibitors (SGLT-2is) and glucagon-like peptide-1 receptor agonists (GLP-1RAs) in patients with type 2 diabetes: a systematic review and network meta-analysis study protocol. BMJ Open 2018;8:e023206. doi:10.1136/ bmjopen-2018-023206

- Prepublication history and additional material for this paper are available online. To view these files, please visit the journal online (http://dx.doi. org/10.1136/bmjopen-2018023206).

Received 26 March 2018 Revised 2 October 2018 Accepted 4 October 2018

\section{Check for updates}

(c) Author(s) (or their employer(s)) 2018. Re-use permitted under CC BY-NC. No commercial re-use. See rights and permissions. Published by BMJ.

${ }^{1}$ Department of Health Sciences, University of Leicester, Leicester, UK

${ }^{2}$ Diabetes Research Centre, Leicester General Hospital, Leicester, UK

${ }^{3} \mathrm{NIHR}$ Leicester Biomedical Research Centre, Leicester, UK

Correspondence to Professor Laura J Gray; Ig48@le.ac.uk

\section{ABSTRACT}

Introduction Sodium-glucose cotransporter 2 inhibitors (SGLT-2is) and glucagon-like peptide-1 receptor agonists (GLP-1RAs) are two classes of glucose-lowering drugs gaining popularity in the treatment of type 2 diabetes mellitus (T2DM). Current guidelines suggest patientcentred approaches when deciding between available hyperglycaemia drugs with no indication to which specific drug should be administered. Despite systematic reviews and meta-analyses being conducted within SGLT-2is and GLP-1RAs, differences across these classes of drugs have not been investigated. Therefore, this systematic review and network meta-analysis (NMA) will aim to compare the efficacy and safety profiles across and within SGLT-2is and GLP-1RAs.

Methods PubMed, the Cochrane Central Register of Controlled Trials and ISI Web of Science will be searched from inception for published randomised controlled trials conducted in patients with T2DM, with at least two arms consisting of SGLT-2is, GLP-1RAs or control/placebo.

Title and abstracts will be screened by two independent reviewers with conflicts resolved by a third. Data will be extracted by the primary researcher, a random sample will be checked by an independent reviewer. Risk of bias will be assessed using the Cochrane Risk of Bias Tool and overall quality of evidence will be assessed using the Grading of Recommendations Assessment, Development and Evaluation approach. Study characteristics, participants baseline characteristics, mean change in cardiometabolic outcomes and number of adverse events will be extracted for each study. Primary outcome will be the mean change in glycated haemoglobin $\left(\mathrm{HbA}_{1 \mathrm{c}}\right)(\%, \mathrm{mmol} / \mathrm{mol})$. Initial randomeffects pairwise meta-analysis will be conducted for each unique treatment comparison where heterogeneity will be assessed. A Bayesian NMA approach will be adopted where random-effects generalised linear models will be fitted in WinBUGS. Sensitivity analysis will be conducted to assess choices of prior distributions and length of burn-in and sample.
Strengths and limitations of this study

- This is the first network meta-analysis to compare efficacy and safety outcomes within and across the two classes of hyperglycaemic drugs: sodium-glucose cotransporter 2 inhibitors and glucagon-like peptide- 1 receptor agonists.

- This study will provide clinically relevant information to clinicians to guide treatment decisions.

- Due to strict inclusion/exclusion criteria, potential selection biases will be minimised.

- There is a possibility of inconsistency between treatment effects which will need to be assessed.

- All outcomes may not be reported in all studies which could lead to the exclusion of drugs in the ranking of treatments for some outcomes.

Ethics and dissemination Ethics approval is not required for this study. Results from this study will be published in a peer-review journal.

PROSPER0 registration number CRD42018091306.

\section{INTRODUCTION}

New drugs for the management of hyperglycaemia in type 2 diabetes mellitus (T2DM) are continuously being developed. In recent years, advancements have been made in two classes of glucose-lowering drug: sodium-glucose cotransporter 2 inhibitors (SGLT-2is) and glucagon-like peptide-1 receptor agonists (GLP-1RAs). Although both classes of drug improve glycaemic control in patients with T2DM, the mechanisms and formulation of these drugs vary, particularly within the class of GLP-1RAs. SGLT-2is improve blood glucose levels by inhibiting glucose reabsorption in the kidneys through SGLT-2 receptors. ${ }^{1}$ The 
formulation of GLP-1RAs differ greatly, with some drugs being exendin- 4 base (eg, exenatide and lixisenatide), while others are formulated as analogues of the human GLP-1 peptide hormone (eg, liraglutide, dulaglutide and semaglutide). ${ }^{2}$ There are further differences within this class of drug, with some being long acting (eg, exenatide once weekly) while others are short acting (eg, exenatide two times per day). ${ }^{2}$

International guidelines recommend SGLT-2is (ie, empagliflozin, canagliflozin, ertugliflozin or dapagliflozin) or GLP-1RAs (ie, exenatide or lixisenatide) as combination therapy with other treatments when monotherapy or dual/triple therapy are not sufficient in reaching the individuals' glycaemic targets. ${ }^{3-6}$ These classes of drugs also improve cardiovascular and renal outcomes, suggesting a patient-centred approach should be undertaken when administering these drugs, depending on patient's health.

Although guidelines have suggested when SGLT-2is and GLP-1RAs should be administered in reducing blood glucose levels, current research lacks clear direction as to which specific drug should be used. In fact, available systematic reviews and network meta-analysis (NMA) have compared effectiveness of these drugs within these two classes $^{7-9}$; however, the benefit-risk profile between the two classes and which drug should be preferred according to background therapy (ie, single, dual or triple failure of glucose-lowering therapy) remains unclear.

\section{Objectives}

This study aims to assess and compare the efficacy and safety profiles across and within two classes of drug, SGLT-2is and GLP-1RAs, in adult ( $\geq 18$ years) patients with T2DM using data from randomised controlled trials (RCTs).

\section{METHODS}

This systematic review and NMA protocol has been developed following the Preferred Reporting Items for Systematic Reviews and Meta-Analyses (PRISMA) Protocols guideline (see online supplementary table 1 ). ${ }^{10}$ The study will follow the PRISMA for Network Meta-Analyses guidelines for reporting the results of the review. ${ }^{11}$

\section{Search strategy}

PubMed, the Cochrane Central Register of Controlled Trials and ISI Web of Science will be searched from inception of the databases to ensure all possible studies on SGLT-2is and GLP-1RAs are included. All databases available in ISI Web of Science will be searched (ie, Web of Science Core Collection, MEDLINE, SciELO, Russian Science Citation Index and KCI-Korean Journal Database). In these databases, restrictions will be placed to exclude case reports, meeting abstracts, reviews, news articles, bibliographies, book chapters, biographies, letters, reference material and editorials.

Key search terms will include all drug names developed within the two drug classes being assessed. These are based on previous systematic reviews conducted in this area. ${ }^{7-9}$ In all database searches, there will be no restrictions on languages. However, once the search has been conducted, papers that are not in English (or have not been translated) will be excluded. Example search strategies for each database are shown in table 1.

\section{Inclusion/exclusion criteria}

Strict inclusion/exclusion criteria have been defined for this study to limit heterogeneity and enhance clinical applicability. Only RCTs will be included in the NMA.

\section{Study population}

Eligible study populations will include adult ( $\geq 18$ years old) patients with T2DM of any duration. Studies conducted in children/adolescents or where all participants included have a specific disease (ie, chronic kidney disease or hypertension) will be excluded. Studies conducted entirely in specific Asian cohorts will be excluded (international studies with centres in Asian countries and other countries will be included) to minimise a systematic bias due to the regular use of lower doses of these drugs in these populations as well as certain drugs being available only in Asian populations.

\section{Table 1 Example search strategies for each database included}

\begin{tabular}{ll}
\hline Database & Example search strategy \\
\hline PubMed & $\begin{array}{l}\text { Exenatide OR Liraglutide OR Lixisenatide OR Albiglutide OR Dulaglutide OR Semaglutide OR } \\
\text { Taspoglutide OR Canagliflozin OR Empagliflozin OR Dapagliflozin OR Ipragliflozin OR Tofogliflozin OR } \\
\text { Luseogliflozin OR Ertugliflozin OR Sotagliflozin OR Efpeglenatide OR (Itca AND 650). }\end{array}$ \\
$\begin{array}{ll}\text { Cochrane Central } \\
\text { Register of }\end{array}$ & $\begin{array}{l}\text { Exenatide OR Liraglutide OR Lixisenatide OR Albiglutide OR Dulaglutide OR Semaglutide OR } \\
\text { Controlled Trials }\end{array}$ \\
& $\begin{array}{l}\text { Taspoglutide OR Canagliflozin OR Empagliflozin OR Dapagliflozin OR Ipragliflozin OR Tofogliflozin OR } \\
\text { variations will be searched). }\end{array}$ \\
ISI Web of Science & TOPIC:(Exenatide) OR TOPIC: (liraglutide) OR TOPIC: (lixisenatide) OR TOPIC:(albiglutide) OR \\
& TOPIC: (dulaglutide) OR TOPIC: (semaglutide) OR TOPIC:(taspoglutide) OR TOPIC:(Canagliflozin) OR \\
& TOPIC:(Empagliflozin) OR TOPIC:(Dapagliflozin) OR TOPIC:(Ipragliflozin) OR TOPIC:(Tofogliflozin) OR \\
& TOPIC:(Luseogliflozin) OR TOPIC:(Ertugliflozin) OR TOPIC:(Sotagliflozin) OR TOPIC:(Efpeglenatide) OR \\
& TOPIC:(Itca AND 650).
\end{tabular}


Intervention(s) and control(s)

Trials should have at least two arms to be included. These could consist of active intervention(s) versus active intervention(s) or active intervention(s) versus control/ placebo. Any single-arm trials will be excluded from the analysis.

The interventions included in this NMA are:

- Any SGLT-2is meeting inclusion criteria given below.

- Any GLP-1RAs meeting inclusion criteria given below.

- Any combination of SGLT-2is and GLP-1RAs.

The $\operatorname{arm}(\mathrm{s})$ with doses recommended by international guidelines and the control/intervention arm(s) will be extracted from the relevant trials for analysis. According to the National Institute for Health and Clinical Excellence and Food and Drug Administration guidelines, the recommended dosage for SGLT-2is and GLP-1RAs are ${ }^{12}$ :

SGLT-2is:

- Canagliflozin: $100 \mathrm{mg}-300 \mathrm{mg}$ once daily

- Empagliflozin: $10 \mathrm{mg}-25 \mathrm{mg}$ once daily

- Dapagliflozin: $5 \mathrm{mg}-10 \mathrm{mg}$ once daily

- Ertugliflozin: $5 \mathrm{mg}-15 \mathrm{mg}$ once daily

GLP-1RAs (long acting):

- Exenatide QW: $2 \mathrm{mg}$ once weekly

- Liraglutide: $1.2 \mathrm{mg}-1.8 \mathrm{mg}$ once daily

- Dulaglutide: $0.75 \mathrm{mg}-1.5 \mathrm{mg}$ once weekly

- Semaglutide: $0.5 \mathrm{mg}-1 \mathrm{mg}$ once weekly

- Albiglutide: $30 \mathrm{mg}-50 \mathrm{mg}$ once weekly GLP-1RAs (short acting):

- Exenatide Twice daily: $5 \mathrm{mcg}-10 \mathrm{mcg}$ twice daily

- Lixisenatide: $10 \mathrm{mcg}-20 \mathrm{mcg}$ once daily

Arms with ipragliflozin, tofogliflozin or luseogliflozin will be excluded as these drugs have approval only in Japan. For drugs that have no recommended dose according to guidelines and have not been excluded, all doses will be extracted. Combination therapy will be allowed if the drug combined with the drug of interest and placebo/control is the same across arms of RCTs initiated prior to baseline.

Although albiglutide and taspoglutide have been withdrawn from the market, these drugs will be included in the NMA as they will contribute to the indirect comparison between drugs included in the network.

The controls included in this NMA will consist of placebo or no treatment given (ie, no other treatment beyond background therapy). Therefore, studies to be included in this analysis will be of the form:

- Intervention(s) versus intervention(s)

- Intervention(s) versus placebo

- Intervention(s) versus no intervention

\section{Primary and secondary outcomes}

The primary outcome will be the mean change in glycated haemoglobin $\left(\mathrm{HbA}_{1 \mathrm{c}}\right)(\%, \mathrm{mmol} / \mathrm{mol})$ at 12 months from baseline. Other cardiometabolic outcomes are the mean change in waist circumference $(\mathrm{cm})$, body weight $(\mathrm{kg})$, total cholesterol $(\mathrm{mmol} / \mathrm{L})$, high-density lipoprotein $(\mathrm{mmol} / \mathrm{L})$ and low-density lipoprotein cholesterol $(\mathrm{mmol} / \mathrm{L})$, triglycerides $(\mathrm{mmol} / \mathrm{L})$, systolic and diastolic blood pressure $(\mathrm{mm} \mathrm{Hg})$, and heart rate (bpm) from baseline. All outcomes will be extracted at approximately 6 and 12 months.

The safety outcomes include the number of patients in each arm to have: a hypoglycaemic event (as defined in the study), diarrhoea, nausea, vomiting, abdominal pain, injection site reaction, amputations, bone fractures, pancreatitis, cancer, diabetic ketoacidosis, genital infection and urinary tract infection. Further, the number of patients tested to be antidrug antibody positive will be assessed.

Studies will be excluded if the primary outcome $\left(\mathrm{HbA}_{1 \mathrm{c}}\right)$ has not been reported.

\section{Study selection}

Once the search has been completed, papers will be imported into EndNote X7.3.1. For each study, the title, authors, year of publication, language of paper, type of publication, journal, volume, issue and pages will be extracted, where duplicate papers across the three database searches will be removed.

Once duplicates have been removed, two independent reviewers will screen titles and abstracts, excluding non-relevant papers according to the inclusion/exclusion criteria. If it is unclear whether a paper has met the inclusion/exclusion criteria, this will be selected for full-text screening. Disagreements on papers for full-text screening between the two reviewers will be resolved by a third reviewer.

Data will then be extracted from relevant papers selected after full-text screening. Additionally, references of included papers will be searched for any other appropriate papers that may have been missed previously. Risk of bias for each trial included will also be assessed. To ensure the quality of extraction, a random sample of $10 \%$ of the included trials will be selected and another reviewer will independently extract data and assess risk of bias. The agreement between primary researcher and the second reviewer will be compared; if the level of agreement is below $80 \%$, a full independent duplicate extraction will be conducted.

\section{Data extraction}

Trial specific data will be extracted using an intention to treat principle, where possible. The duration of the trial (weeks) will be extracted along with the background intervention given to all participants in the study, $\operatorname{drug}(\mathrm{s})$ given in each arm, dosage of the drug and the number of participants recruited to each arm. Pooled mean values (ie, means of all participants combined regardless of treatment) will be extracted for each trial baseline characteristic. Baseline characteristics extracted will include the mean values for age (years), weight $(\mathrm{kg})$, body mass index $\left(\mathrm{kg} / \mathrm{m}^{2}\right)$, percentage of men, $\mathrm{HbA}_{1 \mathrm{c}}(\%, \mathrm{mmol} / \mathrm{mol})$ and diabetes duration (years) at baseline. If the pooled mean is unavailable, using the number of participants recruited to each arm and arm-specific baseline characteristic mean 
values, the pooled average for the whole study population will be calculated using the following equation:

$$
\mu_{\text {pooled }}=\frac{\sum_{i} \mu_{\text {arm } i^{*} n_{i}}}{\sum_{i} n_{i}}
$$

where:

- $n_{i}=$ number of participants in arm $i$

- $\mu_{\text {arm } i}=$ mean value of outcome of interest in arm $i$

For each cardiometabolic outcome of interest, arm-specific mean differences from baseline will be extracted. If this is not provided, the difference in the mean change from baseline between arms in each trial will be extracted. Measures of variability will also be extracted to indicate the uncertainty of the estimates. These include: variances, $\mathrm{SD}, \mathrm{SE}$ or CI, other test statistics and $\mathrm{p}$ values, if available. These will be converted into a consistent measure of variability (SD) before analysis using the Cochrane collaborations guidance. ${ }^{14}$

For each adverse effect, the number of participants in each arm to have at least one event will be extracted.

Data unavailable in the paper (main text and supplementary material) will be supplemented by checking if trials are registered on ClinicalTrials.gov and extracting any study results available. Trials will be excluded for a particular outcome if data are unavailable in the paper and on ClinicalTrials.gov. A table will be produced for the supplementary material, listing each trial and outcomes to show which trials contributed to each outcome analysed.

\section{Meta-bias and risk of bias assessment}

Publication bias will be assessed using funnel plots for comparisons including more than 10 studies. In pairwise meta-analysis, funnel plots are scatter plots of 'study effect sizes against some measure of precision' ${ }^{15}$ However, pairwise meta-analysis consists of only one comparison, while there are multiple comparisons being estimated in an NMA. Therefore, comparison-adjusted funnel plots will be fitted, which is a recently developed methodology available in Stata (StataCorp, College Station, Texas, USA). ${ }^{15}$

The risk of bias will be assessed using the Cochrane risk of bias assessment tool. ${ }^{16}$ The sources of bias assessed will include whether a random sequence generation was used, if treatment allocation was concealed, blinding of participants and researchers from what treatment participants received, incompleteness of any primary outcomes and selectivity of reporting. Each of the sources of bias in each trial will be assessed and classed as either 'high risk', 'low risk' or 'unclear risk', where the percentages for each category in each source of bias analysed will be described and the results interpreted taking the risk of bias into account. Sensitivity analysis will also be conducted excluding studies reporting high risk of bias in any domain analysed.

\section{Strategy for data synthesis}

Initially, pairwise meta-analyses will be conducted for individual comparisons included in this analysis.
Random-effects models will be fitted in Stata, using the metan command, to assess direct comparisons between treatments. Heterogeneity will be assessed by calculating $I^{2}$ values, where larger values indicate higher levels of heterogeneity. If there are high levels of heterogeneity (ie, $I^{2} \geq 75 \%$ ) in pairwise comparisons, it may not be suitable to pool these studies in the NMA.

Network plots will then be produced in Stata, using the network plot command, to visually represent direct and indirect comparisons being calculated for each of the outcomes being assessed in this study. ${ }^{15}$

An NMA will be conducted in WinBUGS using a Bayesian methodology. Random-effects generalised linear models (GLMs), using Markov Chain Monte Carlo simulation method, will be fitted in order to estimate overall treatment effects so that various drugs can be compared and ranked by percentage improvement in accordance to the primary cardiometabolic outcome $\left(\mathrm{HbA}_{1 \mathrm{c}}\right){ }^{17}$ As some studies will have multiple arms, a multivariate normal distribution will be placed on the random-effect terms in order to account for the between-arm correlations. Throughout the analysis, the placebo arm will be included as a single node and will be used as the reference treatment. Meta-regression will be used to account for and explain high levels of heterogeneity by analysing the impact of study level covariates.

For the cardiometabolic outcomes, a normal likelihood will be used along with an identity link function in order to estimate the mean change from baseline for the treatments in comparison to placebo. Adverse events outcomes will be analysed using a logistic regression model (ie, binomial likelihood and logit link function) in order to estimate the odds of events in the treatment groups in comparison to the placebo group.

For each model fitted, initially, a burn-in of 10000 samples will be used along with a simulation run length of 50000 samples. If convergence is not achieved, then these levels will be increased. Vague normal priors will be placed on the trial baseline effects, mean treatment effects and SD. However, sensitivity of priors will be tested by using alternative precisions for baseline effects and mean treatment effects as well as alternative prior distributions, such as the gamma distribution, for SD. Additionally, autocorrelation of chains will be assessed by visually analysing autocorrelation plots. For models with high levels of autocorrelation, the chain will be thinned and the number of samples in the burn-in and simulation will be increased. History plots will be assessed to verify adequate length of burn-in and simulation length. If there is a large amount of deviation in these plots, the burn-in and sample length will be increased. Finally, convergence of each model will be tested using Brooks Gelman-Rubin plots.

To test the assumption of consistency across direct and indirect treatment comparisons, design-by-treatment interaction models described by Higgins et $a l^{18}$ will be used. In order to study if the heterogeneity assumption of NMA holds, $\tau^{2}$ calculations will be conducted which indicates the variability between study effect estimates, with 
larger $\tau^{2}$ values representing higher levels of heterogeneity between studies.

For continuous cardiometabolic outcomes, effect estimates of mean differences along with $95 \%$ credible intervals $(95 \% \mathrm{CrI})$ will be calculated, while for dichotomous variables OR with $95 \%$ CrI. Forest plots will be produced to show the mean differences (or OR) between active treatments in comparison to placebo, while comparison tables will be reported to illustrate the overall effect estimates of comparisons between each treatment (ie, all SGLT-2is, all GLP-1RAs and placebo/control) included in this study. Treatments will be also ranked in accordance to which provided the greatest improvement in $\mathrm{HbA}_{1 \mathrm{c}}(\%$, $\mathrm{mmol} / \mathrm{mol}$ ) from baseline.

Sensitivity analysis will be conducted assessing any changes in effect estimates when varying burn-in and iteration lengths. Further, different priors will be used to study if this will have an effect on the estimates from random-effects NMA models fitted.

Subgroup analysis will be conducted where each NMA will be stratified by therapy failure (ie, drug naive, single, dual or triple failure). This will allow to rank drugs according to the previous treatments.

\section{Overall interpretation (Grading of Recommendations Assessment, Development and Evaluation)}

Overall quality of evidence for each outcome assessed will be rated using an approach developed from the Grading of Recommendations Assessment, Development and Evaluation Working Group methodology. ${ }^{19}$ This approach will assess the quality of evidence for each outcome for direct and indirect estimates as well as rating the overall quality of evidence from the NMA effect estimates.

\section{Ethics and dissemination}

Formal ethics approval is not required for this study as confidential patient data will not be analysed. The results from this study, which is expected to be completed by September 2018, will be submitted to a peer-review journal. The protocol for this NMA has been registered (registration number: CRD42018091306) with PROSPERO (International Prospective Register of Systematic Reviews).

\section{Patient and public involvement}

Patients and public were not involved in the design of this study.

\section{DISCUSSION}

GLP-1RAs and SGLT-2is have gained increasing popularity in the management of hyperglycaemia in T2DM, with additional evidence from studies suggesting benefits in reducing cardiovascular and renal risk. ${ }^{20-24}$ Previous meta-analyses analysing the cardiometabolic and safety aspects within these classes of drugs have been conducted; however, these studies have grouped all drugs within these classes together and have not accounted for the heterogeneity between drugs within the classes. ${ }^{25} 26$ Although improvements have been made in the methods of analysis, with more relevant NMAs published in recent years, ${ }^{7-9}$ guidance of administrating these drugs are limited in terms of specific drug benefits of GLP-1RAs and SGLT-2is with no comparisons made between these classes of drugs.

This systematic review and NMA will be the first to compare treatments for glycaemic control in patients with T2DM across both GLP-1RAs and SGLT-2is; and will assess their comparative effectiveness accounting for current patient therapy. Additionally, this will be the first NMA to include combination of SGLT-2is and GLP-1RAs arms as part of the analysis, allowing an informed decision to be made on whether a combination of the two classes provided a benefit in comparison to single use of the drugs.

A very broad search strategy will be used in this study, which will ensure any trials with indirect comparisons will be included making this study more comprehensive. Due to strict inclusion/exclusion criteria defined, potential biases will be minimised (eg, by excluding studies including only patients with chronic kidney disease, the risk of bias due to the systematic differences of this population will be reduced).

There are also limitations to this study which need to be considered. It may not be possible to extract all cardiometabolic and safety outcomes for each trial included, which will reduce the strength of evidence when analysing these particular outcomes as it is possible that certain comparisons may not be able to be estimated from the network. Inconsistency between treatment effects may arise; however, design-by-treatment interaction models will be used in order to test this assumption. Although inclusion/exclusion criteria has been defined to limit heterogeneity, this may still exist. Therefore, meta-regression will be used to assess the impact of study level covariates in order to minimise this. Finally, sensitivity analysis will need to be conducted in order to show robustness of results as well as the appropriate use of prior distributions when fitting Bayesian random effects GLMs.

\section{CONCLUSION}

To our knowledge, this is the first NMA that will be conducted to compare and rank SGLT-2is and GLP-1RAs for glycaemic control in patients with T2DM and assess their efficacy and safety profile according to therapy failure. These results will be relevant and clinically meaningful to current clinical practice. Further, results will be instrumental for the development of future guidelines and help tailor the treatment to individual needs (ie, stratified/personalised medicine).

Contributors Contributors to the conception, design and/or interpretation of this systematic review and network meta-analysis protocol: HH, LJG, KK, FZ, NND and MJD. Drafting and/or revising protocol for important content: HH, LJG, KK, FZ, NND and MJD. Approving the final version of the protocol: HH, LJG, KK, FZ, NND and MJD. Accountability of all aspects of the work: HH, LJG, KK, FZ, NND and MJD. 
Funding This report is the independent research of $\mathrm{HH}$ supported by the National Institute of Health Research Collaborations for Leadership in Applied Health Research and Care-East Midlands (NIHR CLAHRC-EM) as part of a PhD project. FZ is a Clinical Research Fellow supported by the NIHR CLAHRC-EM.

Competing interests None declared.

Patient consent Not required.

Provenance and peer review Not commissioned; externally peer reviewed.

Open access This is an open access article distributed in accordance with the Creative Commons Attribution Non Commercial (CC BY-NC 4.0) license, which permits others to distribute, remix, adapt, build upon this work non-commercially, and license their derivative works on different terms, provided the original work is properly cited, appropriate credit is given, any changes made indicated, and the use is non-commercial. See: http://creativecommons.org/licenses/by-nc/4.0/.

\section{REFERENCES}

1. Faillie JL. Pharmacological aspects of the safety of gliflozins. Pharmacol Res 2017;118:71-81.

2. Tran KL, Park YI, Pandya S, et al. Overview of glucagon-like peptide-1 receptor agonists for the treatment of patients with type 2 diabetes. Am Health Drug Benefits 2017;10:178.

3. National Institute for Health and Clinical Excellence. The management of type 2 diabetes. 2015. https://www.nice.org.uk/ guidance/ng28 (Accessed 25 Dec 2017).

4. Inzucchi SE, Bergenstal RM, Buse JB, et al. Management of hyperglycemia in type 2 diabetes, 2015: a patient-centered approach: update to a position statement of the American Diabetes Association and the European Association for the Study of Diabetes. Diabetes Care 2015;38:140-9.

5. American Diabetes Association. 8. Pharmacologic approaches to glycemic treatment: standards of medical care in diabetes-2018. Diabetes Care 2018;41:S73-85.

6. Garber AJ, Abrahamson MJ, Barzilay Jl, et al. Consensus statement by the American Association of Clinical Endocrinologists and American College of Endocrinology on the comprehensive type 2 diabetes management algorithm - 2018 executive summary. Endocr Pract 2018;24:91-120.

7. Htike ZZ, Zaccardi F, Papamargaritis D, et al. Efficacy and safety of glucagon-like peptide-1 receptor agonists in type 2 diabetes: a systematic review and mixed-treatment comparison analysis. Diabetes, Obesity and Metabolism 2017;19:524-36.

8. Zaccardi F, Webb DR, Htike ZZ, et al. Efficacy and safety of sodiumglucose co-transporter-2 inhibitors in type 2 diabetes mellitus: systematic review and network meta-analysis. Diabetes Obes Metab 2016;18:783-94.

9. Zaccardi F, Htike ZZ, Webb DR, et al. Benefits and harms of onceweekly glucagon-like peptide-1 receptor agonist treatments: a systematic review and network meta-analysis. Ann Intern Med 2016;164:102-13.
10. Shamseer L, Moher D, Clarke M, et al. Preferred reporting items for systematic review and meta-analysis protocols (PRISMA-P) 2015: elaboration and explanation. BMJ 2015;349:97647.

11. Hutton B, Salanti G, Caldwell DM, et al. The PRISMA extension statement for reporting of systematic reviews incorporating network meta-analyses of health care interventions: checklist and explanations. Ann Intern Med 2015:162:777-84.

12. NHS Health Development Agency, National Institute for Health and Care Excellence (Great Britain). British National Formulary. 2018. https://bnf.nice.org.uk/ (Accessed 15 Jan 2018).

13. Food and Drug Administration, United States. FDA. https://www.fda. gov/ (Accessed 15 Jan 2018).

14. Higgins JP, Green S. Cochrane Handbook for Systematic Reviews of Interventions Version 5.1.0 (updated March 2011): The Cochrane Collaboration. 2011.

15. Chaimani A, Higgins JP, Mavridis D, et al. Graphical tools for network meta-analysis in STATA. PLoS One 2013;8:e76654

16. Higgins JP, Altman DG, Gøtzsche PC, et al. The Cochrane Collaboration's tool for assessing risk of bias in randomised trials. BMJ 2011;343:d5928.

17. Dias $\mathrm{S}$, Sutton AJ, Ades AE, et al. Evidence synthesis for decision making 2: a generalized linear modeling framework for pairwise and network meta-analysis of randomized controlled trials. Med Decis Making 2013;33:607-17.

18. Higgins JP, Jackson D, Barrett JK, et al. Consistency and inconsistency in network meta-analysis: concepts and models for multi-arm studies. Res Synth Methods 2012;3:98-110.

19. Puhan MA, Schünemann HJ, Murad MH, et al. A GRADE Working Group approach for rating the quality of treatment effect estimates from network meta-analysis. BMJ 2014;349:g5630.

20. Holman RR, Bethel MA, Mentz RJ, et al. Effects of once-weekly exenatide on cardiovascular outcomes in type 2 diabetes. $N$ Engl $J$ Med 2017;377:1228-39.

21. Marso SP, Daniels GH, Brown-Frandsen K, et al. Liraglutide and cardiovascular outcomes in type 2 diabetes. N Engl J Med 2016;375:311-22.

22. Zinman B, Wanner C, Lachin JM, et al. Empagliflozin, cardiovascular outcomes, and mortality in type 2 diabetes. N Engl J Med 2015;373:2117-28

23. Bethel MA, Patel RA, Merrill $P$, et al. Cardiovascular outcomes with glucagon-like peptide-1 receptor agonists in patients with type 2 diabetes: a meta-analysis. Lancet Diabetes Endocrinol 2018;6.

24. Zhang XL, Zhu QQ, Chen YH, et al. Cardiovascular safety, longterm noncardiovascular safety, and efficacy of sodium-glucose cotransporter 2 inhibitors in patients with type 2 diabetes mellitus: a systemic review and meta-analysis with trial sequential analysis. $J$ Am Heart Assoc 2018;7:e007165.

25. Karagiannis T, Liakos A, Bekiari E, et al. Efficacy and safety of once-weekly glucagon-like peptide 1 receptor agonists for the management of type 2 diabetes: a systematic review and metaanalysis of randomized controlled trials. Diabetes Obes Metab 2015;17:1065-74.

26. Monami M, Nardini C, Mannucci E. Efficacy and safety of sodium glucose co-transport-2 inhibitors in type 2 diabetes: a meta-analysis of randomized clinical trials. Diabetes Obes Metab 2014;16:457-66. 